\title{
LEVANTAMENTO PRELIMINAR DOS GÊNEROS E ESPÉCIES DE BAETIDAE (INSECTA: EPHEMEROPTERA) DO ESTADO DE SÃO PAULO, COM ÊNFASE EM COLETAS REALIZADAS EM CÓRREGOS FLORESTADOS DE BAIXA ORDEM
}

\author{
Frederico Falcão Salles ${ }^{1,2}$; Cesar N. Francischetti ${ }^{1}$; Fabio de Oliveira Roque ${ }^{3}$; Mateus Pepinellï, Susana Trivinho \\ Strixino $^{3}$
}

Biota Neotropica v3 (n2) http://www.biotaneotropica.org.br/v3n2/pt/abstract?short-communication+BN01103022003

\author{
Recebido em: 12/06/2003 \\ Revisado em: 20/08/2003 \\ Publicado em: 26/08/2003
}
1. Universidade Federal de Viçosa. Museu de Entomologia, Departamento de Biologia Animal, Viçosa, Minas Gerais, Brasil;
2. Autor correspondente. E-mail: ffsalles@insecta.ufv.br
3. Universidade Federal de São Carlos. Laboratório de Entomologia Aquática, Departamento de Hidrobiologia, Universidade Federal de São Carlos. Homepage: www.ufscar.br/ leia

\begin{abstract}
Preliminary survey of genera and species of Baetidae (Insecta: Ephemeroptera) from São Paulo State, with emphasis in the fauna sampled in low order forested stream.

Although in Brazil, there has been an increasing number of studies on Baetidae in the last years, until the present study only three nominal species have been reported from the São Paulo State. Herein, we report the following taxa sampled in 15 low order forested streams from different macro-regions of São Paulo State: Americabaetis alphus, A. labiosus, A. longetron, Apobaetis fiuzai, Aturbina georgei, Baetodes cf. serratus, Callibaetis sp. 1, Callibaetis sp. 2, Camelobaetidius anubis, Cloeodes irvingi, Cryptonympha sp., Paracloeodes eurybranchus, Waltzoyphius fasciatus, and Zelusia principalis.
\end{abstract}

Key words: Baetidae, Ephemeroptera, Brazil, São Paulo State, new reports.

\section{Resumo}

Levantamento preliminar dos gêneros e espécies de Baetidae (Insecta: Ephemeroptera) do Estado de São Paulo, com ênfase em coletas realizadas em córregos florestados de baixa ordem

A despeito dos avanços alcançados recentemente com relação ao conhecimento faunístico de Baetidae no Brasil, assim como na Região Sudeste, até o presente trabalho somente três espécies nominais haviam sido registradas para o Estado de São Paulo. Baseados em coletas realizadas em quinze córregos de baixa ordem localizados em seis macro-regiões do estado, os seguintes táxons de Baetidae são reportados: Americabaetis alphus, A. labiosus, A. longetron, Apobaetis fiuzai, Aturbina georgei, Baetodes cf. serratus, Callibaetis sp. 1, Callibaetis sp. 2, Camelobaetidius anubis, Cloeodes irvingi, Cryptonympha sp., Paracloeodes eurybranchus, Waltzoyphius fasciatus e Zelusia principalis.

Palavras-chave:Baetidae, Ephemeroptera, Brasil, Estado de São Paulo, novos registros. 


\section{Introdução}

O conhecimento faunístico da família Baetidae (Ephemeroptera) no Brasil vem sofrendo significativos avanços nos últimos anos, e como conseqüência disso, o número de gêneros e espécies reportados para o país aumentou consideravelmente nesse período (de quatro gêneros e dezesseis espécies em 1991, para os atuais dezenove e 40, respectivamente). Fazendo uma análise mais apurada desses registros, pode-se verificar que a maioria encontra-se restrita a poucas e isoladas localidades, principalmente no norte e sul do país. Portanto, o conhecimento dessa família de Ephemeroptera em outras regiões e estados brasileiros é praticamente nulo (LugoOrtiz et al. 2002). Mesmo a Região Sudeste, alvo recente de uma série de estudos taxonômicos lidando com a família (e.g. Lugo-Ortiz et al. 2002, Salles \& Lugo-Ortiz 2002, 2003, Salles et al. 2003a,b, Francischetti et al. 2003), encontra-se nessa situação. Como as áreas mais estudadas nessa região estão localizadas em Minas Gerais e no Rio de Janeiro, o que se sabe a respeito dos demais estados ainda se encontra muito aquém do satisfatório. Enquanto os primeiros registros da família foram realizados recentemente no Espírito Santo (Lugo-Ortiz et al. 2002), o Estado de São Paulo, com três espécies descritas no início do século, baseadas somente em adultos [Callibaetis jocosus (Navás), C. zonalis (Navás) e Moribaetis comes (Navás) (Gillies 1990, Lugo-Ortiz \& McCafferty 1999)], não possui nenhuma outra espécie nominal registrada.

Estimulados pela importância e diversidade da família, e objetivando ampliar o conhecimento a respeito do grupo em ambientes lóticos no Estado de São Paulo, apresentamos uma série de registros inéditos de Baetidae para o estado. Baseado em coletas realizadas principalmente em córregos florestados de baixa ordem, este trabalho tem como intuito contribuir com o Projeto "Levantamento da fauna e da biologia de macroinvertebrados de água doce dos principais mananciais do Estado de São Paulo, com ênfase aos bivalves, Insecta (Plecoptera, Trichoptera e Diptera) e Crustacea (Decapoda)", dentro do Programa BIOTA-FAPESP (Fundação de Amparo à Pesquisa do Estado de São Paulo).

\section{Material e Métodos}

O material analisado é referente a coletas realizadas em quinze córregos de baixa ordem localizados em seis macro-regiões do Estado de São Paulo (Fig. 1): Litoral Sul (Cananéia, Parque Estadual da Serra do Mar), Litoral Centro (Cubatão, Parque Estadual da Serra do Mar - Núcleo Cubatão), Litoral Norte (Ubatuba, Parque Estadual da Serra do Mar - Núcleo Picinguaba), Planalto Centro (São Paulo, Parque Estadual do Jaraguá), Planalto Sul (Parque Estadual Intervales) e Interior (Campus UFSCAR, São Carlos). O planejamento amostral, da maior parte dos córregos analisados, seguiu planejamento estratificado adotado pelos projetos de levantamento de Chironomidae (F. O. Roque) e Simuliidae (M. Pepinelli) dentro do Programa BIOTAFAPESP. Na maioria dos córregos foram realizadas coletas em diferentes meso-hábitats (corredeira e remanso) buscando maximizar o esforço amostral e coletar a maior riqueza de táxons possível. A caracterização dos locais de coleta seguiu recomendações descritas na Ficha Padrão do Programa BIOTA-FAPESP.

Nos registros apresentados na seção abaixo, além de comentários referentes a cada espécie, estão presentes apenas o nome do córrego e o número de indivíduos coletados. Informações mais detalhadas sobre os locais de coleta encontram-se inseridas separadamente na Tabela 1. O material encontra-se depositado na coleção do Museu de Zoologia da Universidade de São Paulo, São Paulo, Brasil.

\section{Resultados}

\section{Americabaetis alphus Lugo-Ortiz \& McCafferty}

Ocorrência (n ${ }^{\circ}$ de indivíduos): Córrego das Pedras (2); Córrego Sede (2).

Comentários: A. alphus é uma das espécies de Baetidae com distribuição conhecida mais ampla na América do Sul, estando previamente registrada para Argentina, Bolívia, Chile, Paraguai e para os estados brasileiros de Minas Gerais, Paraná, Rio de Janeiro, Rio Grande do Sul e Santa Catarina (Lugo-Ortiz \& McCafferty 1996c, Francischetti et al. 2003). Dessa forma, sua presença já era esperada no Estado de São Paulo e a espécie provavelmente encontrase distribuída nos demais estados da Região Sudeste. Suas ninfas foram encontradas tanto em áreas de corredeira como de remanso.

\section{Americabaetis labiosus Lugo-Ortiz \& McCafferty}

Ocorrência (n ${ }^{\circ}$ de indivíduos): Córrego Espraiado (1).

Comentários: A. labiosus estava inicialmente registrada para o Paraguai, Uruguai, e no Brasil, para os estados do Paraná, Santa Catarina, Rio Grande do Sul (LugoOrtiz \& McCafferty 1996c). Recentemente, Francischetti et al. (2003), registraram a espécie para o Estado do Rio de Janeiro, o que juntamente com sua distribuição prévia já indicava a sua presença em São Paulo.

\section{Americabaetis longetron Lugo-Ortiz \& McCafferty}

Ocorrência ( $\mathrm{n}^{\circ}$ de indivíduos): Córrego Cambuci (1); Córrego Chefão (1).

Comentários: A. longetron, assim como A. alphus e A. labiosus, encontra-se amplamente distribuída, com registros prévios para o Paraguai, Uruguai, e no Brasil, para 


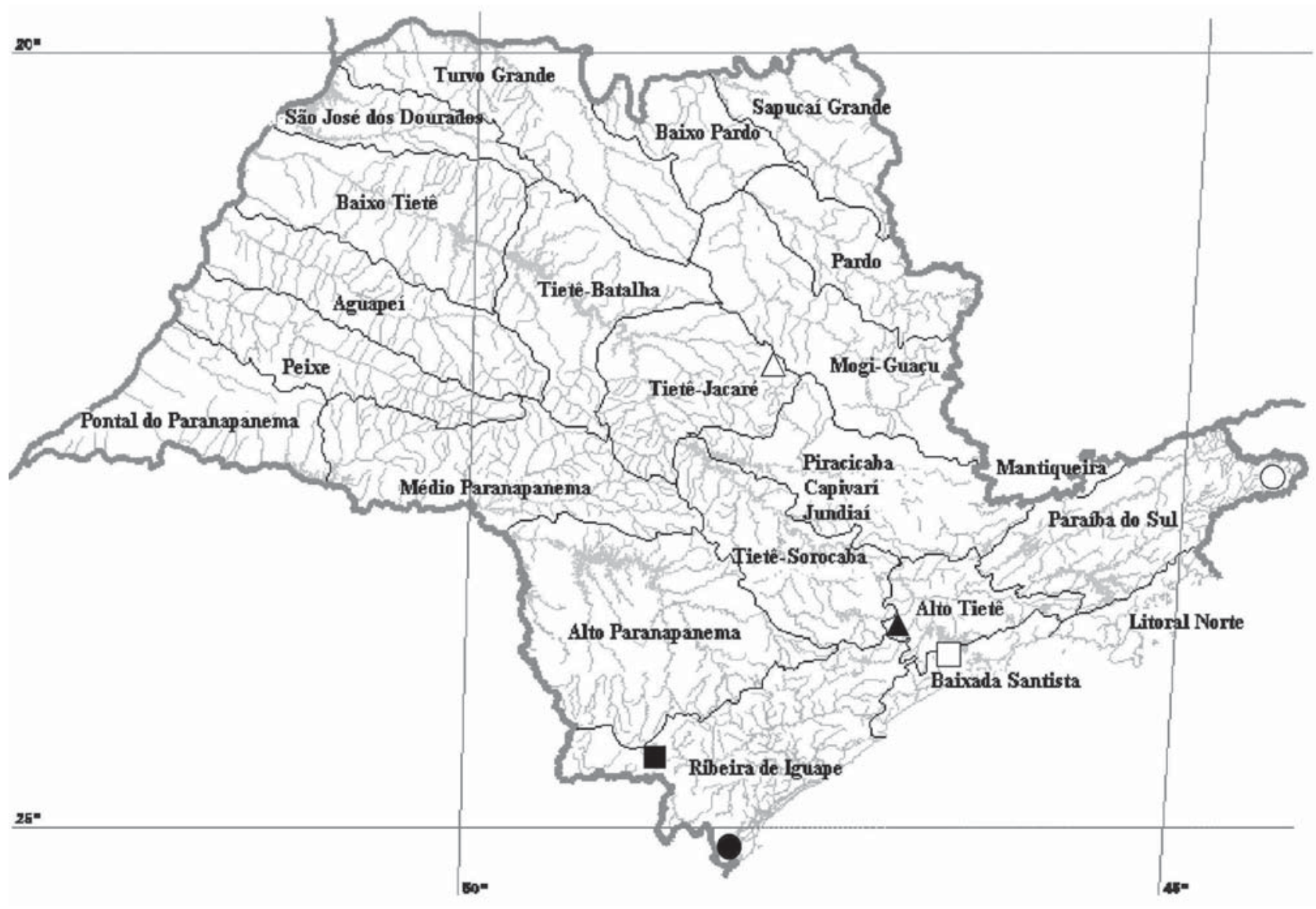

Fig 1. Mapa ilustrativo do Estado de São Paulo com referência às localidades consideradas no presente estudo:

- Cananéia; $\square$ - Parque Estadual de Intervales; $\square$-Cubatão; $\mathbf{O}$ - Ubatuba; $\mathbf{\Delta}$ - São Paulo; $\Delta$-São Carlos. 
Tabela 1. Lista dos córregos onde foram realizadas as coletas, acompanhada de suas coordenadas geográficas, município, bacia hidrográfica, ordem e datas de coleta. (*) Coordenadas referentes ao Parque Estadual do Jaraguá, (**) coordenadas referentes ao Parque Estadual Intervales.

\begin{tabular}{|c|c|c|c|c|c|}
\hline Córrego & $\begin{array}{l}\text { Coordenadas } \\
\text { geográficas }\end{array}$ & Município / Local & $\begin{array}{l}\text { Bacia } \\
\text { Hidrográfica }\end{array}$ & Ordem & $\begin{array}{l}\text { Data da } \\
\text { coleta }\end{array}$ \\
\hline C. da Gruta & $\begin{array}{l}24^{\circ} 53^{\prime} 03,7^{\prime \prime} \mathrm{S} \\
47^{\circ} 51^{\prime} 22,8^{\prime \prime} \mathrm{W}\end{array}$ & Cananéia & Ribeira de Iguape & $1-2$ & $\begin{array}{l}07 / 2001 \\
02 / 2002\end{array}$ \\
\hline C. Chefão & $\begin{array}{l}24^{\circ} 53^{\prime} 02,0^{\prime \prime} \mathrm{S} \\
47^{\circ} 51^{\prime} 22,3^{\prime \prime} \mathrm{W}\end{array}$ & Cananéia & Ribeira de Iguape & $1-2$ & $\begin{array}{l}07 / 2001 \\
02 / 2002\end{array}$ \\
\hline C. Longe & $\begin{array}{l}24^{\circ} 54^{\prime} 12,6^{\prime \prime ~ S} \\
47^{\circ} 58^{\prime} 36,9^{\prime \prime} \mathrm{W}\end{array}$ & Cananéia & Ribeira de Iguape & $1-2$ & $\begin{array}{l}07 / 2001 \\
02 / 2002\end{array}$ \\
\hline C. do Jeep & $\begin{array}{l}23^{\circ} 54^{\prime} 15,2^{\prime \prime} \mathrm{S} \\
46^{\circ} 28^{\prime} 46,9^{\prime \prime} \mathrm{W}\end{array}$ & $\begin{array}{l}\text { Cubatão / Núcleo } \\
\text { Cubatão }\end{array}$ & Baixada Santista & $1-2$ & $09 / 2001$ \\
\hline C. Cambuci & $\begin{array}{l}23^{\circ} 54^{\prime} 08^{\prime \prime} \mathrm{S} \\
46^{\circ} 28^{\prime} 23,2^{\prime \prime} \mathrm{W}\end{array}$ & $\begin{array}{l}\text { Cubatão / Núcleo } \\
\text { Cubatão }\end{array}$ & Baixada Santista & $1-2$ & $09 / 2001$ \\
\hline C. da Sede & $\begin{array}{l}23^{\circ} 21^{\prime} 50,2^{\prime \prime} \mathrm{S} \\
44^{\circ} 49^{\prime} 12,8^{\prime \prime} \mathrm{W}\end{array}$ & $\begin{array}{l}\text { Ubatuba / Núcleo } \\
\text { Picinguaba }\end{array}$ & Litoral Norte & $1-2$ & $\begin{array}{l}09 / 2001 \\
03 / 2002\end{array}$ \\
\hline C. da Vespa & $\begin{array}{l}23^{\circ} 20^{\prime} 15,7^{\prime \prime} \mathrm{S} \\
44^{\circ} 50^{\prime} 14,6^{\prime \prime} \mathrm{W}\end{array}$ & $\begin{array}{l}\text { Ubatuba / Núcleo } \\
\text { Picinguaba }\end{array}$ & Litoral Norte & $1-2$ & $\begin{array}{l}09 / 2001 \\
03 / 2002\end{array}$ \\
\hline C. Canabineiros & $\begin{array}{l}23^{\circ} 24^{\prime} \mathrm{S} \\
45^{\circ} 44^{\prime} \mathrm{W} *\end{array}$ & $\begin{array}{l}\text { São Paulo / P. E. } \\
\text { Jaraguá }\end{array}$ & Alto Tietê & $1-2$ & $07 / 2001$ \\
\hline C. das Pedras & $\begin{array}{l}23^{\circ} 24^{\prime} \mathrm{S} \\
45^{\circ} 44^{\prime} \mathrm{W} *\end{array}$ & $\begin{array}{l}\text { São Paulo / P. E. } \\
\text { Jaraguá }\end{array}$ & Alto Tietê & $1-2$ & $\begin{array}{l}09 / 1998 \\
07 / 2001\end{array}$ \\
\hline C. do Silêncio & $\begin{array}{l}23^{\circ} 24^{\prime} \mathrm{S} \\
45^{\circ} 44^{\prime} \mathrm{W} *\end{array}$ & $\begin{array}{l}\text { São Paulo / P. E. } \\
\text { Jaraguá }\end{array}$ & Alto Tietê & $1-2$ & $07 / 2001$ \\
\hline C. do Fazzari & $\begin{array}{l}21^{\circ} 58^{\prime} 07,6^{\prime \prime} \mathrm{S} \\
47^{\circ} 53^{\prime} 08,8^{\prime \prime} \mathrm{W}\end{array}$ & São Carlos & Tietê -Jacaré & $1-2$ & $\begin{array}{l}06 / 2001 \\
04 / 2002\end{array}$ \\
\hline C. Espraiado & $\begin{array}{l}21^{\circ} 53^{\prime} 50 ”, \mathrm{~S} \\
47^{\circ} 52^{\prime} 25^{\prime \prime} \mathrm{W}\end{array}$ & São Carlos & Tietê -Jacaré & $1-2$ & $05 / 2002$ \\
\hline C. Bocaína & $\begin{array}{l}24^{\circ} 16^{\prime} 13^{\prime \prime} \mathrm{S} 48^{\circ} 27^{\prime} 9^{\prime \prime} \\
\mathrm{W}\end{array}$ & Ribeirão Grande & Ribeira de Iguape & $1-2$ & $\begin{array}{l}08 / 2001 \\
02 / 2002\end{array}$ \\
\hline $\begin{array}{l}\text { C. Água } \\
\text { Comprida }\end{array}$ & $\begin{array}{l}24^{\circ} 12^{\prime}-24^{\circ} 32^{\prime \prime} \mathrm{S} \\
48^{\circ} 03^{\prime}-48^{\circ} 32^{\prime} \mathrm{W} * *\end{array}$ & Ribeirão Grande & Ribeira de Iguape & $1-2$ & $\begin{array}{l}08 / 2001 \\
02 / 2002\end{array}$ \\
\hline Rio das Mortes & $\begin{array}{l}\text { S } 24^{\circ} 20^{\prime} 20,2^{\prime \prime} \\
48^{\circ} 26^{\prime} 03,2^{\prime \prime} \mathrm{W} \\
\end{array}$ & Ribeirão Grande & Ribeira de Iguape & $3-4$ & $\begin{array}{l}08 / 2001 \\
02 / 2002 \\
\end{array}$ \\
\hline
\end{tabular}

Obs: os nomes dos córregos foram atribuídos pelos autores. 
os estados do Espírito Santo, Minas Gerais, Paraná, Rio de Janeiro e Santa Catarina (Lugo-Ortiz \& McCafferty 1996c, Lugo-Ortiz et al., 2002, Francischetti et al. 2003). As ninfas foram coletadas em áreas de corredeira e remanso. De acordo com Lugo-Ortiz et al. (2002), provavelmente a espécie seja tolerante a altos níveis de poluição orgânica.

\section{Apobaetis fiuzai Salles \& Lugo-Ortiz}

Ocorrência ( $\mathrm{n}^{\circ}$ de indivíduos): Córrego da Gruta (1).

Comentários: A. fiuzai foi descrita recentemente para os estados de Mato Grosso e Minas Gerais (Salles \& LugoOrtiz 2002), e apesar de somente uma ninfa ter sido coletada em remanso, seu meso-hábitat condiz com o reportado originalmente para a espécie. O novo registro estende ao sul a distribuição da espécie.

\section{Aturbina georgei Lugo-Ortiz \& McCafferty}

Ocorrência ( $\mathrm{n}^{\circ}$ de indivíduos): Córrego Longe (1); Córrego da Vespa (1).

Comentários: A. georgei estava previamente registrada para a Guiana Francesa, o Paraguai e Brasil, estados do Amazonas, Mato Grosso e Pará (Lugo-Ortiz \& McCafferty 1996a). Além de ser o primeiro registro da espécie para toda a Região Sudeste, sua presença no município de Cananéia e Ubatuba, litoral do estado (Fig. 1), estende consideravelmente a leste a sua distribuição. As duas ninfas coletadas foram encontradas em áreas de remanso.

\section{Baetodes cf. serratus Needham \& Murphy}

Ocorrência (n ${ }^{\circ}$ de indivíduos): Córrego da Cachoeira da Água Comprida (7); Córrego Bocaina (2); Rio das Mortes (19); Córrego Longe (3).

Comentários: B. serratus, espécie tipo do gênero, foi descrita baseada em ninfas coletadas no município do Rio de Janeiro (Needham \& Murphy 1924). Posteriormente, Traver (1944) registrou a espécie para Minas Gerais e desde então nenhuma outra citação foi feita a seu respeito. Como de costume para as ninfas do gênero, todos os espécimes de $B$. cf. serratus foram coletados em área de corredeira. Como a descrição da espécie é antiga e baseada em apenas um indivíduo, sua identificação mais precisa só será possível a partir de um estudo mais detalhado.

\section{Callibaetis sp. 1 e Callibaetis sp. 2}

Ocorrência ( $\mathrm{n}^{\circ}$ de indivíduos): Córrego Canabineiros (1); Córrego Sede (2).

Comentários: Único dos gêneros encontrados neste trabalho previamente reportado para o estado, Callibaetis Eaton é um dos raros casos de Baetidae presentes na América do Sul cuja taxonomia é baseada principalmente no estágio alado. Isto dificulta imensamente a identificação específica de seus representantes no estágio ninfal. Além de $C$. jocosus e $C$. zonalis, três outras espécies já haviam sido registradas para o estado, porém baseadas em ninfas e não denominadas (Traver 1944, Demoulin 1955). Em função da dificuldade do gênero, não foi possível associar as ninfas coletadas no presente estudo com as previamente registradas para o estado. Callibaetis é típico de ambientes lênticos, podendo contudo ser encontrado em áreas de remanso de sistemas lóticos, como foi o caso das ninfas coletadas.

\section{Camelobaetidius anubis (Traver \& Edmunds)}

Ocorrência ( $\mathrm{n}^{\circ}$ de indivíduos): Rio das Mortes (2).

Comentários: A espécie e o gênero são pela primeira vez registrados para a Região Sudeste. C. anubis apresentava a sua distribuição restrita à Região Sul do Brasil, nos estados do Paraná e Santa Catarina (Traver \& Edmunds 1968).

\section{Cloeodes irvingi Waltz \& McCafferty}

Ocorrência ( $\mathrm{n}^{\circ}$ de indivíduos): Córrego da Cachoeira da Água Comprida (3); Córrego Jeep (1); Córrego Longe (1); Córrego das Pedras (1); Córrego Vespa (1).

Comentários: $C$. irvingi foi recentemente registrada para o Brasil, Estado do Espírito Santo (Lugo-Ortiz et al. 2002), e até então a espécie só havia sido registrada para a localidade-tipo, no Paraguai (Waltz \& McCafferty 1987). Dessa forma, sua presença no Estado de São Paulo preenche uma lacuna com relação à sua distribuição. Suas ninfas foram coletadas em pequena quantidade em áreas de corredeira e de remanso.

\section{Cryptonympha sp.}

Ocorrência ( ${ }^{\circ}$ de indivíduos): Córrego Espraiado (10); Córrego Longe (1).

Comentários: O gênero Cryptonympha Lugo-Ortiz \& McCafferty é composto apenas por uma espécie descrita, C. copiosa Lugo-Ortiz \& McCafferty, com ampla distribuição no Brasil (estados do Acre, Amazonas, Pará, Rio Grande do Sul e Santa Catarina) (Lugo-Ortiz \& McCafferty 1998). Além de C. copiosa, Roback (1966) e Orth et al. (2000) reportaram duas espécies não identificadas para o Peru e Guiana Francesa, respectivamente. As ninfas coletadas no presente trabalho tratam-se de fato de uma nova espécie, e sua descrição encontra-se em preparação. O gênero é portanto registrado pela primeira vez para toda a Região Sudeste. Todos os espécimes foram coligidos em correnteza. 


\section{Paracloeodes eurybranchus Lugo-Ortiz \& McCafferty \\ Ocorrência ( $\mathrm{n}^{\circ}$ de indivíduos): Córrego Espraiado (1); Córrego Gruta (2). \\ Comentários: P. eurybranchus estava inicialmente registrada para o Brasil (Estado do Rio Grande do Sul) e norte da Argentina (Lugo-Ortiz \& McCafferty 1996b). Posteriormente, Lugo-Ortiz et al. (2002) a reportaram para o Estado do Espírito Santo, estendendo consideravelmente ao norte sua distribuição. A presença da espécie no Estado de São Paulo era portanto esperada. De acordo com Lugo- Ortiz et al. (2002) a espécie é possivelmente tolerante à poluição orgânica. As ninfas foram coletadas tanto em áreas de corredeira como remanso.}

\section{Waltzoyphius fasciatus McCafferty \& Lugo-Ortiz} (27).

Ocorrência ( $\mathrm{n}^{\circ}$ de indivíduos): Córrego Espraiado

Comentários: Descrita originalmente para o Paraguai e Brasil (estados do Amazonas e Pará) (Lugo-Ortiz \& McCafferty 1995) e posteriormente registrada para o Espírito Santo (Lugo-Ortiz et al. 2002), W. fasciatus é uma espécie com ampla distribuição no Brasil e provavelmente na América do Sul. Suas ninfas foram coletadas em áreas de corredeira, juntamente com as de Cryptonympha sp.

\section{Zelusia principalis Lugo-Ortiz \& McCafferty}

Ocorrência ( $\mathrm{n}^{\circ}$ de indivíduos): Córrego Chefão (8); Córrego Espraiado (1); Córrego Fazzari (1); Córrego Jeep (3); Córrego Longe (2); Córrego Pedras (10); Córrego Silêncio (2); Córrego Vespa (3).

Comentários: Mais uma espécie de ampla distribuição, Z. principalis foi inicialmente registrada para o Brasil, estados do Amazonas e Pará, para a fronteira do Brasil com o Suriname e Colômbia (Lugo-Ortiz \& McCafferty 1998), e posteriormente para o Estado do Espírito Santo (Lugo-Ortiz et al. 2002). Z. principalis foi coletada tanto em áreas de corredeira como de remanso, sendo neste estudo a espécie encontrada na maior variedade de córregos.

\section{Discussão}

Foram encontrados ao todo onze gêneros e treze espécies de Baetidae nos quinze riachos estudados. Adicionando esses novos registros às espécies nomeadas, previamente reportadas para o estado (Callibaetis jocosus, C. zonalis e Moribaetis comes), São Paulo passa a contar com um número de registros superior ao dos demais estados brasileiros. Esses novos registros demonstram claramente o quão incipiente é o conhecimento relativo à fauna do grupo no Brasil. Vale ainda ressaltar, que as coletas foram realizadas basicamente em córregos de baixa ordem e localizados nas regiões central e litorânea do estado (Fig. 1), de forma que à medida que novas coletas forem feitas, não só em rios de média e alta ordem, como também em áreas mais a leste do São Paulo, esse valor provavelmente se mostrará superior.

\section{Agradecimentos}

À Fundação de Coordenação de Aperfeiçoamento de Pessoal de Nível Superior (CAPES; Brasília, Brasil) por prover fundos para FFS como estudante de pós-graduação na Universidade Federal de Viçosa, MG. Este estudo foi financiado pela Fundação de Amparo a Pesquisa do Estado de São Paulo, dentro do Programa Biota FAPESP. Gostaríamos de agradecer todos os nossos amigos que nos auxiliaram no trabalho de campo.

\section{Referências bibliográficas}

DEMOULIN, G. 1955. Une mission biologique belge au Brésil. Éphéméroptères. Bull. Inst. R. Sci. Nat. Belg., 31(20): 132.

FRANCISCHETTI, C.N., SALLES, F.F., DA-SILVA, E.R. \& LUGO-ORTIZ, C.R. 2003. First report of Americabaetis Kluge (Ephemeroptera: Baetidae) from Rio de Janeiro, Brazil. Entomotropica, 18(1): 69-71.

GILLIES, M.T. 1990. A revision of the Argentine species of Callibaetis Eaton (Baetidae: Ephemeroptera). Revta Soc. ent. argent. 48(1-4): 15-39.

LUGO-ORTIZ, C.R. \& McCAFFERTY, W.P. 1995. Three distinctive new genera of Baetidae (Insecta: Ephemeroptera) from South America. Annls Limnol, 31(4):233-243.

LUGO-ORTIZ, C.R. \& McCAFFERTY, W.P. 1996a. Aturbina georgei gen et sp-n. A small minnow mayfly (Ephemeroptera, Baetidae) without turbinate eyes. Aquat. Insects, 18:175-183.

LUGO-ORTIZ, C.R. \& McCAFFERTY, W.P. 1996b. The genus Paracloeodes (Insecta, Ephemeroptera, Baetidae), and its presence in South America. Annls Limnol, 32(3):161-169

LUGO-ORTIZ, C.R. \& McCAFFERTY, W.P. 1996c. Taxonomy of the Neotropical genus Americabaetis, new status (Insecta: Ephemeroptera Baetidae). Stud. Neotrop. Fauna Environ., 31:156-169.

LUGO-ORTIZ, C.R. \& McCAFFERTY, W.P. 1998. Five new genera of Baetidae (Insecta: Ephemeroptera) from South America. Annls Limnol, 34(1):57-73.

LUGO-ORTIZ, C.R. \& McCAFFERTY, W.P. 1999. Revision of the south american species of Baetidae (Ephemeroptera) previously placed in Baetis Leach and Pseudocloeon Klapálek. Annls Limnol., 35: 257-262. 
LUGO-ORTIZ, C.R., SALLES, F.F. \& FURIERI, K.S. 2002. First records of small minnow mayflies (Ephemeroptera: Baetidae) from the state of Espírito Santo, southeastern Brazil. Lundiana, 3: 79-80.

NEEDHAM, J.G. \& MURPHY, H.E. 1924. Neotropical mayflies. Bull. Lloyd Library Botany, Pharmacy Materia Medica No 24, Entomol. Ser., 4:1-79.

ORTH, K., THOMAS, A.G.B., DAUTA, C., HOREAU, V., BROSSE, S. \& ADEMMER, C. 2000. Les Ephémères de la Guyane Francaise. 1. Premier inventaire générique, à but de biosurveillance [Ephemeroptera]. Ephemera, 2: 25-38.

ROBACK, S.S. The Catherwood Foundation Peruvian-Amazon Expedition. VI. Ephemeroptera nymphs. Monogr. Acad. Nat. Sci. Philadelphia. 14:129-199.

SALLES, F.F. \& LUGO-ORTIZ, C.R. 2002. A distinctive new species of Apobaetis (Ephemeroptera: Baetidae) from Mato Grosso and Minas Gerais, Brazil. Zootaxa, 35: 1-6.

SALLES, F.F. \& LUGO-ORTIZ, C.R. 2003. Um novo gênero e espécie de Baetidae (Ephemeroptera) do estado de Minas Gerais, Sudeste do Brasil. Iheringia, Sér. Zool., 93(2): 201206.

SALLES, F.F, LUGO-ORTIZ, C.R, DA-SILVA, E.R. \& FRANCISCHETTI, C.N. 2003a. Novo gênero e espécie de Baetidae (Insecta: Ephemeroptera) do Brasil. Arq. Mus. Nac., 61: 23-30.

SALLES, F.F, DA-SILVA, E.R. \& LUGO-ORTIZ, C.R. 2003 b. Descrição da ninfa e redescrição dos adultos de Callibaetis radiatus Navás (Insecta: Ephemeroptera: Baetidae). Lundiana, 4(1): 13-18.

TRAVER, J.R. 1944. Notes on Brazilian mayflies. Bol. Mus. Nac., N.S., Zool., 22:2-53.

TRAVER, J.R. \& EDMUNDS JR, G.F., 1968. A revision of the Baetidae with spatulate-clawed nymphs (Ephemeroptera). Pac. Insects, 10: 629-677.

WALTZ, R.D. \& McCAFERTY, W.P. 1987. Revision of the genus Cloeodes Traver (Ephemeroptera: Baetidae). Ann. Entomol. Soc. Amer., 80: 191-207.

Título: Levantamento preliminar dos gêneros e espécies de Baetidae (Insecta: Ephemeroptera) do Estado de São Paulo, com ênfase em coletas realizadas em córregos florestados de baixa ordem

Autores: Frederico Falcão Salles; Cesar N. Francischetti; Fabio de Oliveira Roque; Mateus Pepinelli, Susana Trivinho Strixino

Biota Neotropica, Vol. 3 ( número 2): 2003 http://www.biotaneotropica.org.br/v3n2/pt/ abstract?short-communication+BN01103022003

Recebido em: 12/06/2003

Revisado em: 20/08/2003

Publicado em: 26/08/2003

ISSN 1676-0603 